\title{
Insights into the mechanism of atrial tachycardia with over two types of reentrant circuits: the important role of the convertibility of functional conduction block regions in maintaining multiple reentrant circuit pathways
}

\author{
Fan Wang ${ }^{1}$, Bai Wang ${ }^{1}$, Jinjin Cui ${ }^{1}$, Ying $\operatorname{Luan}^{1}$, Jian $\mathrm{Xu}^{1}$, Wei Cao ${ }^{1}$, Zhilin Yue ${ }^{1}$, shuo \\ zhang ${ }^{1}$, and Shufeng $\mathrm{Li}^{1}$ \\ ${ }^{1}$ Second Affiliated Hospital of Harbin Medical University
}

January 17, 2021

\begin{abstract}
Background: Multiple atrial tachycardias (ATs) in one patient usually require more complex ablation procedures. Despite the superior accuracy and understanding of conduction features provided by high-resolution mapping, Multiple ATs are still associated with high recurrence rates, and other mechanisms may play a role. Therefore, we aimed to uncover the substrates maintaining these multiple reentrant circuits and the probable mechanisms for the high occurrence of arrhythmia. Methods: Mapping via the Carto system was carried out in 8 patients with more than two types of reentrant circuits during ablation. Functional conduction block (FCB) regions were marked and further analyzed. Results: Twenty sustained ATs were mapped in the 8 patients. Five of these patients exhibited a potential FCB region that changed between different ATs. The potentials of these regions converted between double potentials (DPs), fractionated potentials (FPs) and normal potential due to the different ATs. The FCB regions were the main obstacles and the center of the reentrant circuit in 8 of 14 ATs, and in the other ATs, these regions played a role in reorganizing the conduction pathway. In the activation mapping, the FCB areas were never the target ablation site. Conclusion: The potential FCB region is common in ATs with more than two types of reentrant circuits, especially in scar-related localized reentry. The convertibility of FCB regions provide one of the critical substrates in maintaining multiple ATs. The changefulness of this substrate may be one of the important causes of the high recurrence of related ATs
\end{abstract}

\section{Introduction}

Multiple atrial tachycardias (ATs) in a patient related to spontaneous polytropic atrial scars or radical atrial fibrillation $(\mathrm{AF})$ ablation are usually characterized by multiple mechanisms and require complex ablation procedures. ${ }^{1}$ Scar-related electrically silent areas (ESAs) and slow conduction to the isthmus are considered two of the main mechanisms of these ATs. Despite targeted ablation of these mechanisms and the use of high-resolution mapping, which provides superior accuracy and understanding of the conduction features, these ATs are associated with high recurrence. ${ }^{1,2}$ ATs in these patients may be associated with more complex substrates and other potential mechanisms. The phenomenon of a functional block has been used to explain some complex sustained arrhythmias. ${ }^{3}$ ATs sustained by a functional block line have been seldom reported. ${ }^{4}$ In the present study, we assessed the characteristics of the electrophysiological mechanism in a series of 8 patients with multiple ATs, and we present the role of the functional block region in this specific category of AT.

\section{Methods}

Patient Characteristics 
A cohort of 65 consecutive patients with AT/flutter who were referred to our institution for radiofrequency (RF) catheter ablation between April 2018 and December 2020 were evaluated retrospectively. Of these 65 patients, a total of 8 patients ( 5 men and 3 women) with more than two types of stable ATs during ablation were included in this study. The patients with unstable cycle length (CL), unsustainable ATs and in whom ablation failed were excluded due to the unascertainable mechanism of these ATs. Seven patients had permanent AT, and 1 patient had paroxysmal AT for an average of 12 months (range, 1 month to 36 months) despite the use of antiarrhythmic drugs. All patients had been on oral anticoagulants before ablation. Transesophageal echocardiography was performed to rule out left atrial (LA) thrombi in all patients. Oral anticoagulants were stopped at admission and replaced with intravenous heparin to maintain partial thromboplastin time at 2 to 3 times the control value. This was stopped 6 to $8 \mathrm{hr}$ before ablation for transseptal puncture. All patients gave written informed consent, and the study protocol was approved by the local Research and Human Ethics Committees.

Electrophysiological Study and Mapping

Antiarrhythmic drugs (except amiodarone) were withdrawn at least 5 half-lives before the procedures; amiodarone was interrupted one month before the procedure. A deflectable decapolar catheter (Dynamic XT, Boston Scientific) was positioned within the coronary sinus. If the patient was in sinus rhythm at the start of the procedure, burst pacing was performed to induce AT. Atrial mapping was performed with a Navistar Thermocool 3.5-mm D-F curve ablation catheter with Smart Touch technology (Biosense Webster) or multipolar mapping catheters (Pentaray, Biosense Webster Inc.). Bipolar electrograms filtered at 10-400 Hz were recorded. A bipolar signal from the coronary sinus (CS) electrode was used as the timing reference. The anatomy of the atrium was reconstructed, and zones of ESAs and double potentials (DPs) were marked. The following thresholds were used to define a scar: scar, $<0.05 \mathrm{mV}$; scar border zone, between 0.05 and 0.5 $\mathrm{mV}$; and normal, $0.5 \mathrm{mV}$. DP was defined as a pair of widely split high-frequency potentials separated by a minimum interval of 30 msec. After sequential construction of the map, all points were manually checked and corrected if necessary, especially for activation timing of local fragmented EGMs. A new map was generated to identify a different reentrant circuit if the P-wave morphology or tachycardia CL changed during RF ablation. A functional conduction block (FCB) region was defined as an area showing a conduction block in one AT but not in another AT or in sinus rhythm (Figs. 1-3). The conduction block line was shown as a white line instead of the conduction time interval between two neighboring tissues over $20 \%$ of the CL in the Carto system. Entrainment mapping was not performed systematically to avoid terminating or transforming tachycardia. This approach was used only when it was suspected that the ATs were related to epicardial conduction.

\section{Definitions of a Reentrant Circuit and Classification of AT}

ATs are classified according to the activation pattern: focal AT, anatomical macroreentrant AT (AMAT), and non-AMAT (localized reentry). As previously reported, ${ }^{1}$ AMATs include peritricuspid flutter (PTF) dependent on the cavotricuspid isthmus (CTI), perimitral flutter (PMF) dependent on the mitral isthmus, and roof-dependent macroreentrant tachycardia (RMAT) dependent on the left atrial roof. Localized scarrelated reentry was defined by atrial activation that was recorded over the entire AT CL of a localized area. The localized reentry circuit was variable in size and was composed of a scar with a conduction block and a channel of slow conduction consistent with an "isthmus." Focal AT was defined as atrial activity originating from a discrete site activating the surrounding tissue centrifugally (not necessarily recorded over the entire AT CL). Multiple-loop ATs were defined as those composed of more than one independent active circuit sharing one common isthmus, including dual-loop ATs and triple-loop ATs, with the entire CL included in each of the circuits. ${ }^{5}$ The epicardial conduction of a CS/vein of Marshall (VOM) system-related AT was suspected if a PMF used only the CS as an epicardial conduction route and the activation map demonstrated a continuous jump over a line of complete block on the mitral isthmus between the distal CS and the left lateral LA and the postpacing interval minus tachycardia cycle length (PPI-TCL) $<20 \mathrm{~ms}$ was confirmed at the circuit. When the PMF used the VOM $(+/-\mathrm{CS})$, the activation map showed a jump between the inferolateral LA (overlying the CS) and/or the mid-to-distal CS region and the left lateral ridge or posterior 
base of the left atrial appendage (LAA), with $>10 \%$ of the TCL missing. Entrainment mapping showed a PPI-TCL [?] $20 \mathrm{~ms}$ at the VOM, the ridge left pulmonary vein (LPV)-LAA, the anterior wall of the LA, and 6 to 11 o'clock on the mitral annulus (MA) and a PPI-TCL $>20 \mathrm{~ms}$ at the distal CS, the posterior wall of the LA and the mitral isthmus. Non-AMAT (localized reentry) around the LAA-LPVs using the $\mathrm{CS} / \mathrm{VOM}$ system demonstrated $>10 \%$ TCL missing around the CS/VOM distribution. A PPI-TCL $<20 \mathrm{~ms}$ was observed at the left lateral ridge, posterior base of the LAA, inferolateral LA or the mid-to-distal CS (ostium of VOM), and a PPI-TCL $>20$ ms was observed in the septal MA. ${ }^{6,7}$

\section{Catheter Ablation}

The appropriate ablation site was determined based on anatomical length, catheter stability, tissue thickness and nearby vulnerable structures. In most cases, this represented the narrowest bridge of conducting tissue between scars or anatomical obstacles or the site of the reentry circuit exhibiting low-voltage fractionated potentials (FPs) occurring in the diastolic phase in relation to the activation of the surrounding myocardium. RF energy was delivered using an irrigated 3.5-mm-tip ablation catheter (Thermocool@ ST catheter, Biosense Webster), with a power of $25-45 \mathrm{~W}$ and a cutoff temperature of $45^{\circ} \mathrm{C}$. The endpoint of the procedure was defined as termination of all AT forms and their noninducibility with incremental and programmed atrial pacing. If a new AT was induced either during ablation or during reinduction, it was targeted for ablation using the same strategy as detailed above.

Postprocedure Management and Follow-up

Patients were monitored overnight and then discharged with instructions to continue the anticoagulant and rhythm-control medications that they had been taking before the procedure. After 3 months, treatment with rhythm-control agents was discontinued in cases with no recurrence of arrhythmia or arrhythmia-related symptoms. Additionally, warfarin or dabigatran was discontinued, and aspirin was substituted if appropriate. All patients were seen at 3,6, and 12 months postablation in the outpatient clinic. At each visit, the patients were investigated with a 24-h Holter monitor and echocardiography. In the case of symptoms, an additional 12-lead electrocardiogram (ECG) was acquired.

Statistical Analysis

All continuous variables are expressed as the mean $\pm \mathrm{SD}$ or median $(25 \%, 75 \%$ interquartile range) where applicable. Statistical differences were evaluated using an ANOVA test. $\mathrm{P}<0.05$ was considered statistically significant. Statistical testing was performed with SAS version 9.2

\section{Results}

\section{Patient Characteristics}

The baseline characteristics of the 8 included patients are summarized in Table 1. Multiple reentrant circuits occurred both in patients with a postablation history (6 patients) and those without a history of previous surgical or catheter intervention (2 patients). One patient had dilated cardiomyopathy with left bundle branch block (LBBB) and was treated with CRT-D. One patient experienced ablation of the CTI due to typical atrial flutter. Five patients experienced AF ablation, and the other 2 patients had no obvious structural heart disease detectable by echocardiography and no history of previous surgical or catheter intervention. Six patients experienced AF ablation without any fragmented EGM-based ablation.

\section{Mapping Results}

The AT mapping results are shown in Table 2. A total of 21 sustained ATs were mapped in the 8 patients. Four patients presented 2 different ATs during ablation, 3 patients presented 3 different ATs during ablation, and 1 patient presented 4 different ATs during ablation. In 7 patients, every AT converted to another one after ablation, and each one was shown to be a new kind of AT. The ATs in one patient (the $7^{\text {th }}$ patient) were suspected to be related to VOM. The different activation mapping results were suspicious due to the activation direction changes by the block of ablation lines and perhaps represented the same reentry through different activation modes. The final entrainment mapping features and ablation site termination 
AT coincided with VOM-related AT, as previously described (Fig. 3) ${ }^{6,7}$ In the first mapping, 4 patients showed the type of localized ATs related to scars, 3 patients showed MAT perimitral, and 1 patient showed CTI-dependent AT. In the second type of AT, 3 patients showed localized ATs related to scars, 4 patients showed MAT, and 1 patient showed focal AT-related scars. Four patients had the third AT, 1 had localized ATs, 2 had RMAT and 1 had focal AT.

\section{Characteristics of the Arrhythmogenic Substrate and the Potential FCB Region}

ATs in 7 of 8 originating patients were associated with the presence of one or more scars. The electrical activity in scar areas always showed DPs, FPs or ESAs. Some scars formed a spontaneous conduction block line or perhaps slow conduction. In these serial cases, we found that some spontaneous lines were not constantly blocked, and some were exhibited only in one AT, which suggests that the conduction characteristics in this region were changed depending on the different mechanisms of ATs (Figs. 1-3). Five of these 8 patients exhibited these potential FCBs, and all of these FCB areas seemed to be near the scar areas. The presentation of the FCB region was not related to the CL of ATs or the mechanism of ATs. The potential of these regions could also be converted between DPs, FPs and normal potential (but showed low amplitude in some cases) due to the different ATs. Three of the others did not show potential FCBs, two were focal mechanisms, and the other one was a double-loop localized reentry, which was performed using a protected channel between an anterior line of DP and an anterior ESA (Fig. 4). ATs maintained by the FCB region are common in localized reentrant ATs. The FCB region was related to damage to the previous ablation line in one patient. The voltage maps of 2 patients were performed during sinus rhythm after ablation, and the potential of the FCB area presented no obvious difference from the adjacent normal area.

\section{RF Ablation}

The ablation strategy was adopted depending on the activation mapping result. Localized reentry aimed to find the isthmus region, which was always located between 2 anatomic barriers, consisting of either the natural MA or pulmonary vein (PV) or acquired ESAs or late diastolic potentials (LDPs) and showed fragmented potentials. All the ATs of localized reentry converted to another AT after fragmented potential ablation. Remapping of the new tachycardia showed that an isthmus, which had been a bystander in the previous tachycardia, had become a critical part in the new circuit in 2 patients (Fig. 1C). The AMATs ablated the narrowest bridge of conducting tissue between scars or anatomical obstacles. The 4 perimitral flutter circuits were ablated by a line connecting the MA to the left superior PV $(n=3)$ and the right superior PV $(n=1)$. The peri-roof circuits were ablated by joining the PV to the contralateral superior PV across the LA roof $(\mathrm{n}=2)$. CTI was ablated in 2 patients due to the CTI-dependent AT in the first mapping. The FCB areas were never the target ablation site after mapping the mechanism of the ATs, but some of the suited ablation sites could be near the barrier formed by them (Fig. 2). No patients received further substrate-modified ablation in AT or sinus rhythm.

Follow-Up

There were no procedure-related complications. All 8 patients were in sinus rhythm after ablation. Despite high-rate pacing, 6 of them were noninducible; atypical nonsustained flutter was inducible in 1, and AF was inducible in 1. A pacemaker was implanted in 1 patient due to sinus bradycardia 3 days after ablation. With a mean follow-up of 12 months, all 7 patients were successfully treated with no antiarrhythmic drugs. LA flutter reoccurred in 1 patient 1 week after ablation.

\section{Discussion}

\section{Main Findings}

Convertibility of the FCB regions occurred in 5 of 8 patients in our study, suggesting that it may not be an uncommon electrophysiological feature in ATs, especially scar-related ATs. This substrate species could be described as exhibiting DPs or fractionated and complex potentials and showed a conduction block through this area in one AT but not in another AT or in sinus rhythm. In 8 of 14 ATs, the FCB regions were the main obstacle and the center of the reentrant circuit (such as in Fig. 1 AT1, Fig. 2 AT2). Other FCB 
regions may participate in reorganizing the conduction pathway maintaining ATs (such as in Fig. 2 AT3, Fig. 3 AT2). A specific FCB region acts as an obstacle only for its corresponding AT and does not seem to play a substantial role in other ATs. In severe native scars or progressed scars related to myocardium disease, the electrophysiology coupled with the complex substrate has not been summarized systematically. The FCB region is occasionally described in atrial-related arrhythmia, and although it is thought to play an important role in some types of arrhythmia, there has been no method of analyzing this mechanism and definitively determining its role. ${ }^{4}$ Our study is the first to describe the convertibility of FCB regions and their important role in maintaining some stable ATs. The instability of the FCB region may be the reason for the high recurrence of related ATs, but this possibility has been shown in only one specific AT and is not easy to identify with conventional mapping procedures. The potential FCB region described in our study may account for another mechanism of multiple ATs.

The Role of the Potential FCB Region in ATs

This is the first study to describe this phenomenon in multiple ATs, mainly because various AT alternates provide the opportunity to show the different conduction features of the potential FCB region under different circumstances. Other scar-related ATs may also exist in the potential FCB region, but only one reentrant circuit cannot show them visually. Ja1s et al. ${ }^{8}$ previously noted the spontaneous zones of blocks in mapping at a series of scar-related ATs. Because no second AT occurred, no description of the changes in these zones of the block under different circumstances is possible. Some rare case reports have shown that a functional block line can sustain a reentrant pathway in a single AT episode and does not exhibit conduction block during coronary sinus pacing. We conclude first that the FCB region functions as an important obstacle that could be circled (such as mitral tricuspids in traditional perimitral reentrant circuits), which is usually the localized reentrant circuit. Second, since this region usually presented in MAT, it may play a role in reorganizing the conduction pathway and increasing the region conduction time that maintains the reentrant circuit. In multiple ATs, the FCB region guided the conduction orientation forming the reentrant circuit in one AT, while after ablation, its reconduction guided another AT circuit. Different from the isthmus, these regions are often not the target ablation site in any of the ATs which is observed or not.

The Location of the Potential FCB Region

Critical areas of arrhythmogenesis often occur in diseased tissue with low-voltage areas that indicate the scarring zone. The potential in scar areas could be recorded as ESAs, LDPs or FPs, each of which acts instead of a different mechanism on arrhythmias. ${ }^{8-11}$ One AT that converted to another kind of AT during ablation could occur due to various causes (abnormal automaticity, triggered activity or reentry). The FCB region we described in this study was more common with scar-related reentry and often could be found near the scarring zone. While the potential of these regions could convert between DPs, FPs and normal potential formation in the different ATs, it was still low voltage compared with that of normal tissue in some ATs or sinus rhythms. This electrophysiological feature in large part may be due to scarring of the atrium due to various heart diseases, while normal tissue could not be seen. Second, except for the effect of the scarring, the regions where FCBs occurred were observed more commonly in the junction between the left atrial appendage and anterior wall of the left atrium (in 3) and the junction between the roof and atrium of the left superior PV (in 2), where Bachmann's bundle is located. ${ }^{12}$ These positions are associated with fiber crisscrosses that may exhibit more electric anisotropy. The scarring may affect the homogeneity of conduction in one orientation during ATs. In patients with previous AF ablation, the first MAT may be due to the circumferential pulmonary vein isolation (CPVI), and the FCB would be related to damage to the previous ablation line.

Insight into the Role of the Potential FCB Region in Other Atrial-related Arrhythmias

In addition to the types of ATs we mentioned, the mechanism of another kind of AT with CL alternans is also related to the FCB, which is a rare phenomenon. ${ }^{13-15}$ In one type, CL alternans resulted from an intermittent 2:1 conduction block within the channel of the small circuit. The other type, the alternate block and conduction of the 2 channels with different velocities, results in CL alternans. ${ }^{13}$ The FCB channel 
of these ATs usually occurs with severe atrial scarring of different causes (prior cardiac surgery, catheter ablation of AF or fibrotic atrial cardiomyopathy). Similar to our study, the FCB region often occurs near the scar area. This may be another expression form of the FCB region.

The DP region means a smaller block area than some of the silent areas because the still potential could be recorded bilaterally. Most DPs or fragmented potentials are more common in AT or AF than in sinus rhythm because the bilateral scar tissue that is recognized in ATs is excited at the same time in sinus rhythm due to the normal conduction pattern, not with a certain direction as atrial flutter (AFL). As we observed in this study, the FCB region participated and maintained the reentrant circuit at different CLs or pathways (Figs. 1 and 2). This phenomenon suggests that some AF patients who still experience recurrence of AF after PVI ablation may also have neglected FCBs, while they have normal conduction substrates after mapping under sinus rhythm. The FCB may maintain the AF under some conditions. More electric anisotropy is related to a more uncertain conduction mode, which may lead to AF. Recently, it has been shown that the ablation of Marshall veins or Bachmann's bundle may further advance the success rate of $\mathrm{AF},{ }^{12,16}$ not only for the trigger in these regions but also for reducing the connection with the atrium that decreases the electric anisotropy of conduction. Judging the electric anisotropy region, such as the FCB, may provide new insight into the formulation of the AF ablation strategy.

ATs with over two types of reentrant circuits are often associated with more complex ablation procedures. ${ }^{1}$ The ablation strategy in our study still depended on the activation mapping results. Despite careful mapping, the reentrant circuit of the second AT could be forecasted before the first ablation in only 1 patient with a double-loop localized reentry who did not show the FCB region (Fig. 4). The instability of the functional zone made the second or third AT unpredictable (Figs. 1 and 2). The occurrence of FCB may depend on the circle length of the ATs, and entrainment mapping may transform tachycardia, confusing the mechanism of ATs. Substrate mapping is also limited by different potentials under different conditions and does not seem to make much sense in optimal ablation strategies. Even though an effective method for identifying a potential FCB region is not yet available, the ablation strategy for addressing this specific substrate is controversial. A future computer simulation may well be used to identify the necessary conditions for FCB and an intervention strategy.

Limitations

This study has several limitations: (1) The patients included in this retrospective study were a highly selected group referred for RF catheter ablation, and the number of patients was limited. (2) The absence of systematic pacing and entrainment because of the risk of inducing AF is a limitation in this study, and the FCB depends on the circuit length of the ATs. Entrainment mapping may transform tachycardias and confuse the understanding of the mechanisms of ATs. (3) We did not perform direct epicardial mapping in patients with a suspected epicardial connection, and therefore, our understanding of these potential connections and the relation with the FCB region remains speculative. (4) The voltage mapping in the Carto system was limited in the present FCB because this region often showed DPs or FPs, of which thresholds often fell to the normal or scar border zone and were not distinguishable from other tissues. (5) While the FCB region is important in maintaining reentry as an obstacle, there is still a lack of effective measures to recognize it without a particular AT episode. Although the FCB was related to scarring of the atrium but not the target ablation site under activation mapping, substrate ablation as a strategy aimed at the FCB region to avoid recurrence of other ATs still needs careful deliberation.

\section{Conclusions}

The potential FCB region is common in ATs with over two types of reentrant circuits, especially in scarrelated localized reentry. The occurrence of this substrate may be due to the native scars or progressed scars of myocardium disease. This substrate species could exhibit DPs or fractionated and complex potentials and showed conduction block through this area in one AT but not in another AT or in sinus rhythm. The FCB region maintains reentrant circuits as an obstacle, and the changefulness of this substrate may be one of the important reasons for the high recurrence of related ATs. 


\section{Acknowledgements}

No

\section{References}

1. Derval N, Takigawa M, Frontera A, Mahida S, Konstantinos V, Denis A, Duchateau J, Pillois X, Yamashita $\mathrm{S}$, Berte $\mathrm{B}$, et al. Characterization of complex atrial tachycardia in patients with previous atrial interventions using high-resolution mapping. JACC Clin Electrophysiol . 2020;6:815-826.

2. Laţcu DG, Bun SS, Viera F, Delassi T, El Jamili M, Al Amoura A, Saoudi N. Selection of critical isthmus in scar-related atrial tachycardia using a new automated ultrahigh resolution mapping system.Circ Arrhythm Electrophysiol . 2017;10:e04510.

3. Ciaccio EJ, Coromilas J, Wit AL, Peters NS, Garan H. Source-sink mismatch causing functional conduction block in re-entrant ventricular tachycardia. JACC Clin Electrophysiol . 2018;4:1-16.

4. Miyazaki S, Hasegawa K, Kaseno K, Tada H. Protected channels can be formed by a functional line of block in human atrial tachycardia. Heart Rhythm . 2019;16:642-643.

5. Takigawa M, Derval N, Maury P, Martin R, Denis A, Miyazaki S, Yamashita S, Frontera A, Vlachos $\mathrm{K}$, Kitamura T, et al. Comprehensive multicenter study of the common isthmus in post-atrial fibrillation ablation multiple-loop atrial tachycardia. Circ Arrhythm Electrophysiol . 2018;11:e006019.

6. Vlachos K, Denis A, Takigawa M, Kitamura T, Martin CA, Frontera A, Martin R, Bazoukis G, Bourier F, Cheniti G, et al. The role of Marshall bundle epicardial connections in atrial tachycardias after atrial fibrillation ablation. Heart Rhythm . 2019;16:1341-1347.

7. Takigawa M, Derval N, Martin CA, Vlachos K, Denis A, Nakatani Y, Kitamura T, Cheniti G, Bourier F, Lam A, et al. Mechanism of recurrence of atrial tachycardia: comparison between first versus redo procedures in a high-resolution mapping system. Circ Arrhythm Electrophysiol . 2020;13:e007273.

8. Jaïs P, Shah DC, Haïssaguerre M, Hocini M, Peng JT, Takahashi A, Garrigue S, Le Métayer P, Clémenty J. Mapping and ablation of left atrial flutters. Circulation . 2000;101:2928-2934.

9. Ouyang F, Ernst S, Vogtmann T, Goya M, Volkmer M, Schaumann A, Bänsch D, Antz M, Kuck KH. Characterization of reentrant circuits in left atrial macroreentrant tachycardia: critical isthmus block can prevent atrial tachycardia recurrence. Circulation . 2002;105:1934-1942.

10. Zhang J, Tang C, Zhang Y, Han H, Li Z, Su X. Electroanatomic characterization and ablation outcome of nonlesion related left atrial macroreentrant tachycardia in patients without obvious structural heart disease. J Cardiovasc Electrophysiol . 2013;24:53-59.

11. Fukamizu S, Sakurada H, Hayashi T, Hojo R, Komiyama K, Tanabe Y, Tejima T, Nishizaki M, Kobayashi Y, Hiraoka M. Macroreentrant atrial tachycardia in patients without previous atrial surgery or catheter ablation: clinical and electrophysiological characteristics of scar-related left atrial anterior wall reentry. $J$ Cardiovasc Electrophysiol . 2013;24:404-412.

12. van Campenhout MJ, Yaksh A, Kik C, de Jaegere PP, Ho SY, Allessie MA, de Groot NM. Bachmann's bundle: a key player in the development of atrial fibrillation? Circ Arrhythm Electrophysiol . 2013;6:10411046 .

13. Zhang J, Zheng L, Zhou D, Zhao A, Tang C, Zhang Y, Su X. Insight Into the mechanism of macroreentrant atrial tachycardia with cycle length alternans using ultrahigh density mapping system. Circ Arrhythm Electrophysiol . 2019;12:e007634.

14. Zhou D, Hu W, Yang G, Chen H, Zhang B, Han J, Yang J, Li L, Zheng L. A postsurgery atrial tachycardia with alternating cycle length: the possible circuits revealed by high-resolution mapping. HeartRhythm Case Rep . 2020;6:297-299. 
15. Takigawa M, Denis A, Vlachos K, Martin CA, Jais P, Derval N. Two consecutive ATs demonstrating a centrifugal pattern; What is the mechanism? J Cardiovasc Electrophysiol . 2019;30:978-980.

16. Valderrábano M, Peterson LE, Swarup V, Schurmann PA, Makkar A, Doshi RN, DeLurgio D, Athill CA, Ellenbogen KA, Natale A, et al. Effect of catheter ablation with vein of marshall ethanol infusion vs catheter ablation alone on persistent atrial fibrillation: the VENUS randomized clinical trial. JAMA . 2020;324:1620-1628.

Tables

Table 1. Clinical and Electrophysiological Data

\begin{tabular}{lllllll}
\hline Patient & Age, $\mathrm{y}$ & Sex & Heart Disease & LA Size, mm & $\begin{array}{l}\text { Ejection } \\
\text { Fraction, \% }\end{array}$ & $\begin{array}{l}\text { Prior Ablation } \\
\text { Procedures }\end{array}$ \\
\hline 1 & 78 & Male & DCM, & 38.1 & 37 & No \\
2 & & LBBB & & & No \\
3 & 37 & Male & non & 38.6 & 52.4 & CPVI, Roof \\
4 & 53 & Male & AF, HF, SD & 45.4 & 35 & line \\
5 & 54 & Female & AF & 45 & 42 & CPVI \\
6 & 67 & Female & AF & 49 & 38 & CPVI \\
7 & 38 & Male & AF & 38.9 & 62 & CTI \\
8 & 64 & Male & non & 53.1 & 30.4 & CPVI \\
\hline
\end{tabular}

$\mathrm{AF}=$ atrial fibrillation; $\mathrm{CPVI}=$ circumferential pulmonary vein isolation; $\mathrm{HF}=$ heart failure; $\mathrm{LBBB}=$ left bundle branch block;

$\mathrm{SN}=$ sinus node disease

Table 2. Mapping Data of Tachycardia

\begin{tabular}{|c|c|c|c|c|c|c|c|c|}
\hline Patient & Morph & $\begin{array}{l}\text { Type of } \\
\text { ATs }\end{array}$ & $\begin{array}{l}\text { AT CL } \\
\text { ms }\end{array}$ & $\begin{array}{l}\text { Ablation } \\
\text { site }\end{array}$ & $\begin{array}{l}\text { FCB } \\
\text { location }\end{array}$ & $\begin{array}{l}\text { FCB } \\
\text { potential }\end{array}$ & $\begin{array}{l}\text { Scar } \\
\text { area }\end{array}$ & Outcol \\
\hline 1 & 2 & $\begin{array}{l}1 \text { Localized } \\
2 \text { Localized }\end{array}$ & 190265 & $\begin{array}{l}1 \text { Linear } \\
\text { (ESA - } \\
\text { RPV) } 2 \text { FP } \\
\text { (Anterior } \\
\text { scar) }\end{array}$ & Roof & $1 \mathrm{DP} 2 \mathrm{FP}$ & $\begin{array}{l}\text { Anterior } \\
+ \text { Roof }\end{array}$ & $\mathrm{SR}$ \\
\hline 2 & 2 & $\begin{array}{l}1 \text { Localized } \\
2 \text { RMAT }\end{array}$ & 220300 & $\begin{array}{l}1 \mathrm{FP} \\
\text { (Anterior) } \\
2 \mathrm{FP} \\
\text { (Mitral } \\
\text { isthmus) }\end{array}$ & $\begin{array}{l}\text { Anterior(near } \\
\text { roof) Ante- } \\
\text { rior(near } \\
\text { LAA) }\end{array}$ & $\begin{array}{l}1 \text { DP(roof) } \\
\text { FP(LAA) } 2 \\
\text { FP(roof) } \\
\text { DP(LAA) }\end{array}$ & $\begin{array}{l}\text { Anterior }+ \\
\text { Mitral } \\
\text { isthmus }\end{array}$ & SR \\
\hline 3 & 3 & $\begin{array}{l}1 \text { PMF } 2 \\
\text { Localized } 3 \\
\text { Localized }\end{array}$ & 210215245 & $\begin{array}{l}1 \text { Mitral } \\
\text { isthmus } 2 \\
\mathrm{FP}(\text { near } \\
\text { roof) } 3 \\
\text { near LAA }\end{array}$ & $\begin{array}{l}\text { Anterior(near } \\
\text { LAA) }\end{array}$ & $\begin{array}{l}1 \text { normal } 2 \\
\text { DP } 3 \\
\text { fragmented }\end{array}$ & $\begin{array}{l}\text { Anterior }+ \\
\text { Roof }\end{array}$ & PM \\
\hline
\end{tabular}




\begin{tabular}{|c|c|c|c|c|c|c|c|c|}
\hline Patient & Morph & $\begin{array}{l}\text { Type of } \\
\text { ATs }\end{array}$ & $\begin{array}{l}\text { AT CL } \\
\text { ms }\end{array}$ & $\begin{array}{l}\text { Ablation } \\
\text { site }\end{array}$ & $\begin{array}{l}\text { FCB } \\
\text { location }\end{array}$ & $\begin{array}{l}\text { FCB } \\
\text { potential }\end{array}$ & $\begin{array}{l}\text { Scar } \\
\text { area }\end{array}$ & Outcon \\
\hline 4 & 2 & $\begin{array}{l}1 \text { Localized } \\
2 \text { Localized }\end{array}$ & 230260 & $\begin{array}{l}1 \mathrm{FP} \\
\text { (Anterior } \\
\text { scar) } 2 \mathrm{FP} \\
\text { (Anterior } \\
\text { scar) }\end{array}$ & No & & Anterior & SR \\
\hline 5 & 2 & $\begin{array}{l}1 \text { PTF } 2 \\
\text { Focal }\end{array}$ & 280280 & $\begin{array}{l}1 \text { CTI line } \\
2 \text { Anterior } \\
\text { scar }\end{array}$ & No & & Anterior & $\mathrm{SR}$ \\
\hline 6 & 3 & $\begin{array}{l}1 \text { PMF } 2 \\
\text { RMAT } 3 \\
\text { RMAT }\end{array}$ & 190190220 & $\begin{array}{l}1 \text { Mitral } \\
\text { isthmus } 2 \\
\text { Roof } 3 \mathrm{FP} \\
\text { (roof scar) }\end{array}$ & $\begin{array}{l}\text { Roof (near } \\
\text { RSPV) }\end{array}$ & $\begin{array}{l}1 \text { normal } 2 \\
\text { DP } 3 \text { DP }\end{array}$ & Roof & $\mathrm{SR}$ \\
\hline 7 & 4 & $\begin{array}{l}1 \text { PMF } 2 \\
\text { PMF } 3 \\
\text { RMAT } 4 \\
\text { Localized }\end{array}$ & 220220220 & $\begin{array}{l}1 \text { Mitral } \\
\text { isthmus } 2 \\
\text { Anterior } \\
\text { line } 3 \text { Roof } \\
\text { line } 4 \text { near } \\
\text { LAA }\end{array}$ & Anterior & $\begin{array}{l}1 \text { normal } 2 \\
\text { DP } 3 \text { FP } 4 \\
\text { FP }\end{array}$ & Anterior & SR \\
\hline 8 & 3 & $\begin{array}{l}1 \mathrm{PTF} 2 \\
\text { PMF } 3 \\
\text { Focal }\end{array}$ & 210220250 & $\begin{array}{l}1 \text { CTI line } \\
2 \text { Mitral } \\
\text { isthmus } 3 \\
\text { posterior } \\
\text { wall near } \\
\text { LIPV }\end{array}$ & No & & No & $\mathrm{SR}$ \\
\hline
\end{tabular}

$\mathrm{AT}=$ atrial tachycardia; MAT $=$ macroreentry atrial tachycardia; $\mathrm{CL}=$ cycle length $; \mathrm{CTI}=$ cavotricuspid isthmus; $\mathrm{DP}=$ double potentials; $\mathrm{ESA}=$ electrically silent area; $\mathrm{FCB}=$ functional conduction block; $\mathrm{FP}=$ fragmented potential; $\mathrm{LAA}=$ left atrial appendage; $\mathrm{PM}=$ pace marker; $\mathrm{PMF}=$ perimitral flutter; $\mathrm{PTF}=$ peritricuspid flutter; RPV, right pulmonary vein; RMAT = roof-dependent macroreentrant tachycardia; SR, sinus rhythm

\section{Figure Legends}

Fig. 1. The mapping results of two kinds of ATs and sinus rhythm in patient 2. A, Activation mapping shows a localized reentry run around the obstruct line formed by scars (black box). The DPs are shown near the scar line in the first AT (A 2). B, After ablation of the isthmus in the scars, AT converted to an RMAT depending on the LPV. The location where the DP showed normal potential in the second AT (B 2), and the white block line disappeared due to nearby atrial chronicle activation. There is a new white block line near the LAA (black box) and the location where the FP shown in AT1 (A 3) converted to a DP in AT2 (B 3). C, An FP covering nearly $1 / 3$ of the CL was mapped between the LIPV and the scars in the mitral isthmus, and the AT converted to SR after ablation at this site. D, In sinus rhythm, the positions that showed potential conversion in different ATs showed normal potentials. There were two regions of the $\mathrm{FCB}$ in this patient that functioned in different mechanisms of ATs. AT = atrial tachycardia; $\mathrm{CL}=\mathrm{cycle}$ length; $\mathrm{DP}=$ double potentials; $\mathrm{FCB}=$ functional conduction block; $\mathrm{FP}=$ fragmented potential; $\mathrm{LAA}=$ left atrial appendage; $\mathrm{LPV}=$ left pulmonary vein; $\mathrm{RMAT}=$ roof-dependent macroreentrant tachycardia; $\mathrm{SR}=$ sinus rhythm.

Fig. 2. The mapping results of three kinds of ATs in patient 3. AT1, activation mapping shows that the mechanism of the first was PMF. AT2, the AT1 converted to a localized reentry with multiple breakouts running around the obstruct line (black box) after ablation of the mitral isthmus. A DP showed near the 
white line. AT3, the AT2 converted to a localized reentry running around the LAA after ablation of the upper breakout, and the white block line disappeared, causing near atrial chronicle activation again. The DP converted to the normal potential again. The regions of the FCB were only shown in AT2 and maintained the localized reentrant circuit. There was a constant scar line near the LSPV (white line). AT= atrial tachycardia; $\mathrm{CL}=$ cycle length; $\mathrm{DPs}=$ double potentials; $\mathrm{FCB}=$ functional conduction block; $\mathrm{FP}=$ fragmented potential; $\mathrm{LAA}=$ left atrial appendage; $\mathrm{PMF}=$ perimitral flutter; $\mathrm{LSPV}=$ left superior pulmonary vein.

Fig. 3. The mapping results of four kinds of ATs in patient 7. AT1, activation mapping shows the doubleloop MAT, and entrainment mapping suggests that a better PPI is related to the PMF. AT2, the double-loop MAT converted to PMF after ablation of the mitral isthmus, and an FCB region was present in the anterior wall (black box). A DP showed near the white line but not in AT1. AT3, for failure to block the mitral isthmus through a traditional ablation strategy, an anterior line was ablated as an alternative plan. After ablation, the AT2 converted to RMAT running around the LSPV, and the white block line disappeared, causing nearby atrium chronicle activation again. The DP converted to the normal potential again but with low voltage. AT4, activation mapping showed $26 \%$ of the TCL $(70 \mathrm{~ms})$ missing. PPI-TCL $<20 \mathrm{~ms}$ at the left lateral ridge, posterior base of the LAA. PPI-TCL $>20 \mathrm{~ms}$ in the septal MA and proximal and distal coronary sinus. AT was terminated to sinus rhythm after ablation in the base of the LAA near the left lateral ridge. The regions of the FCB are only shown in AT 2, and the relationship with this AT is not clear. AT= atrial tachycardia; $\mathrm{CL}=$ cycle length; $\mathrm{DP}=$ double potentials; $\mathrm{FCB}=$ functional conduction block; $\mathrm{FP}=$ fragmented potential; $\mathrm{LAA}=$ left atrial appendage; $\mathrm{PMF}=$ perimitral flutter; $\mathrm{LSPV}$, left superior pulmonary vein; $\mathrm{PPI}=$ postpacing interval; TLC $=$ tachycardia cycle length; RMAT = roof-dependent macroreentrant tachycardia.

Fig. 4 . The mapping results of two kinds of ATs in patient 4. AT1, activation mapping shows the doubleloop MAT related to the scar in the anterior wall. There were two breakout pathways of the scar. AT2, after ablation of the lower breakout, the AT1 converted to AT2, which could be forecasted before the first ablation. The AT converted to sinus rhythm after ablation of the upper breakout. No FCB region could be seen during ablation.

\section{Figures}
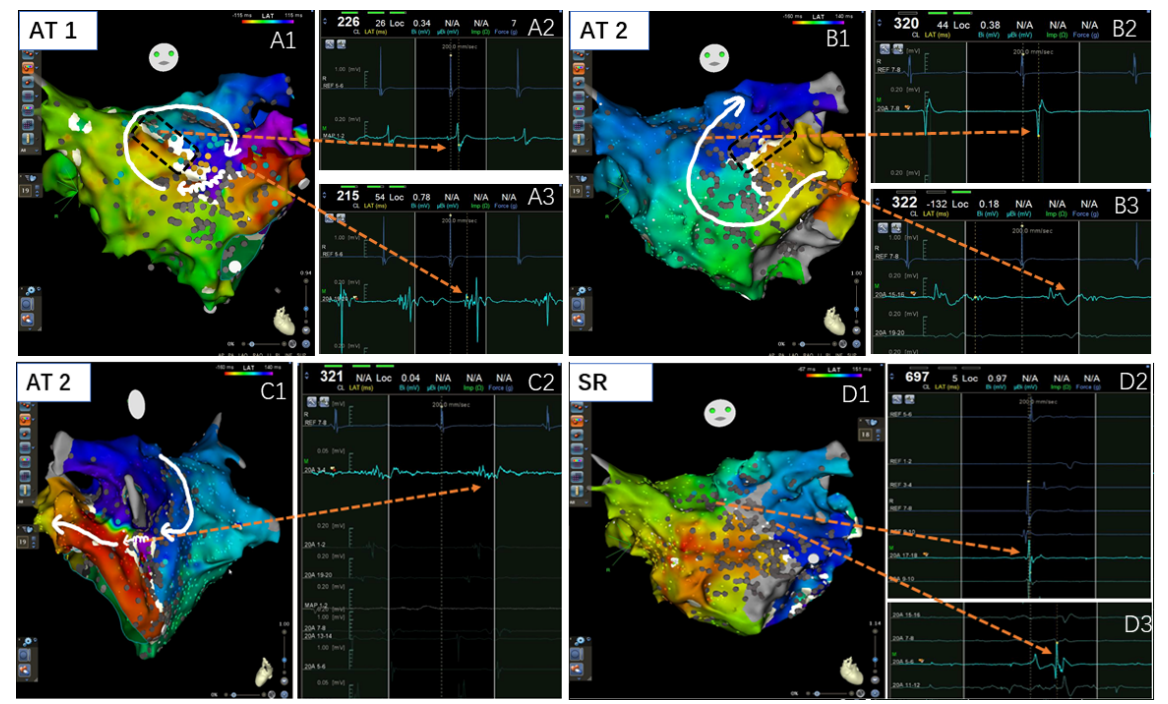

Fig. 1 


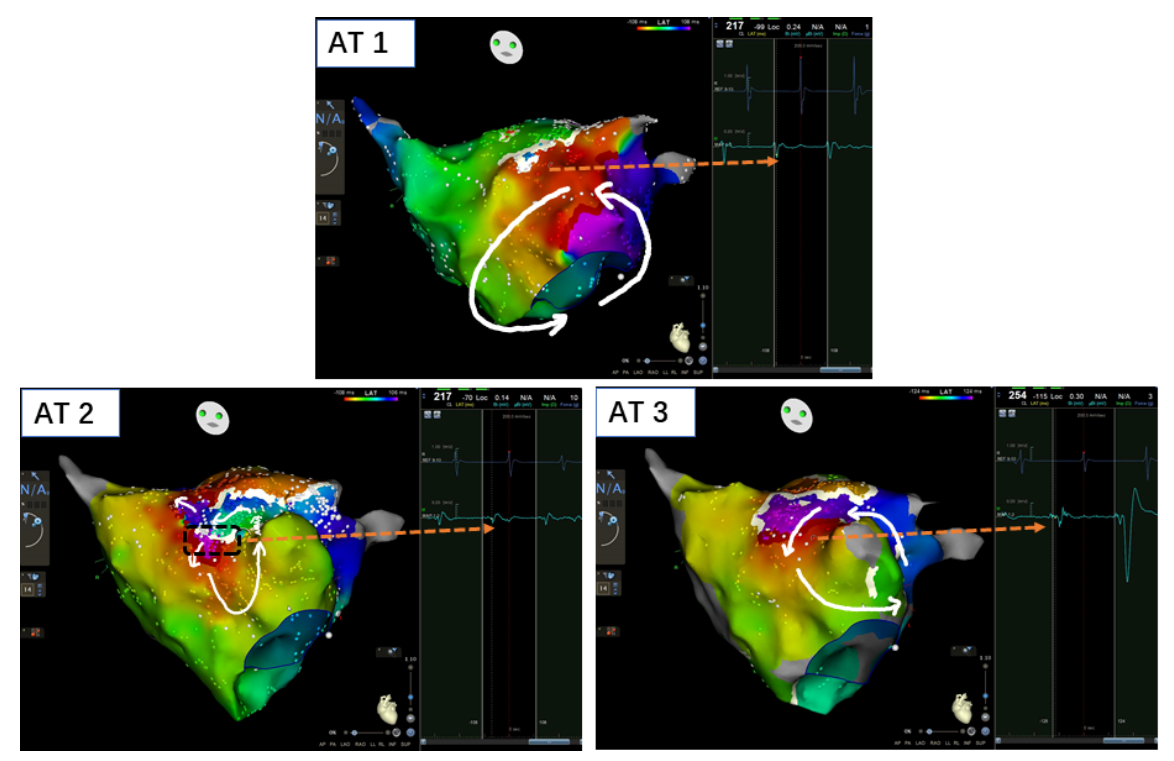

Fig. 2
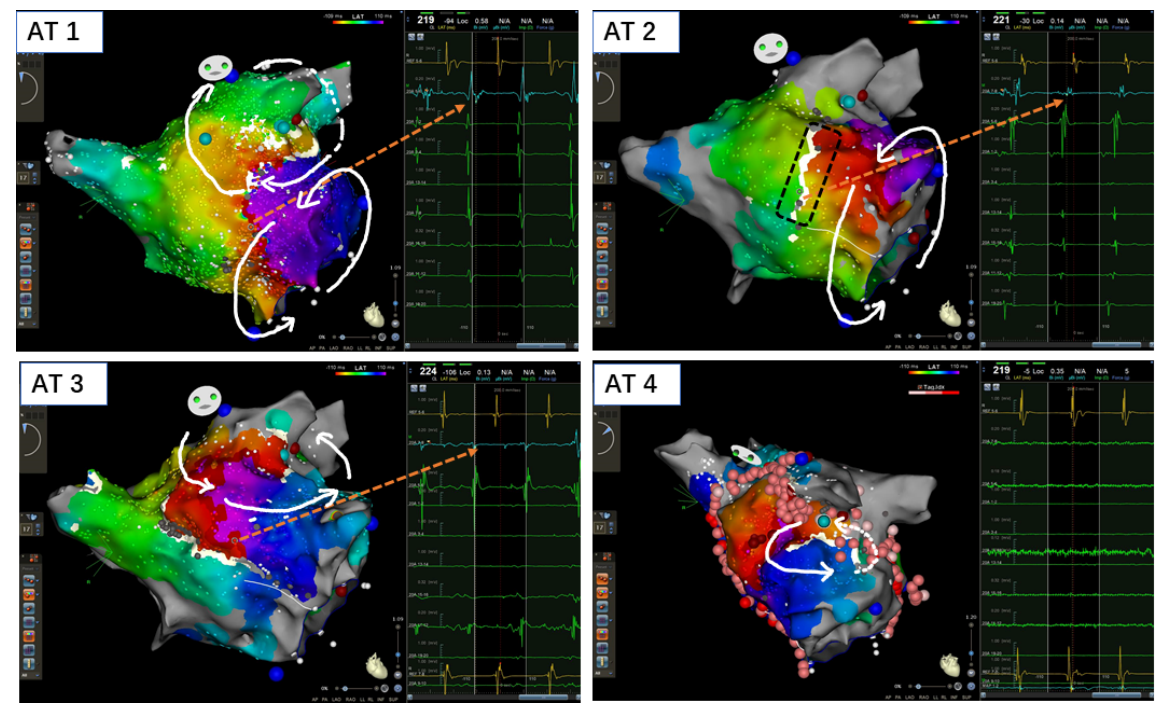

Fig. 3 


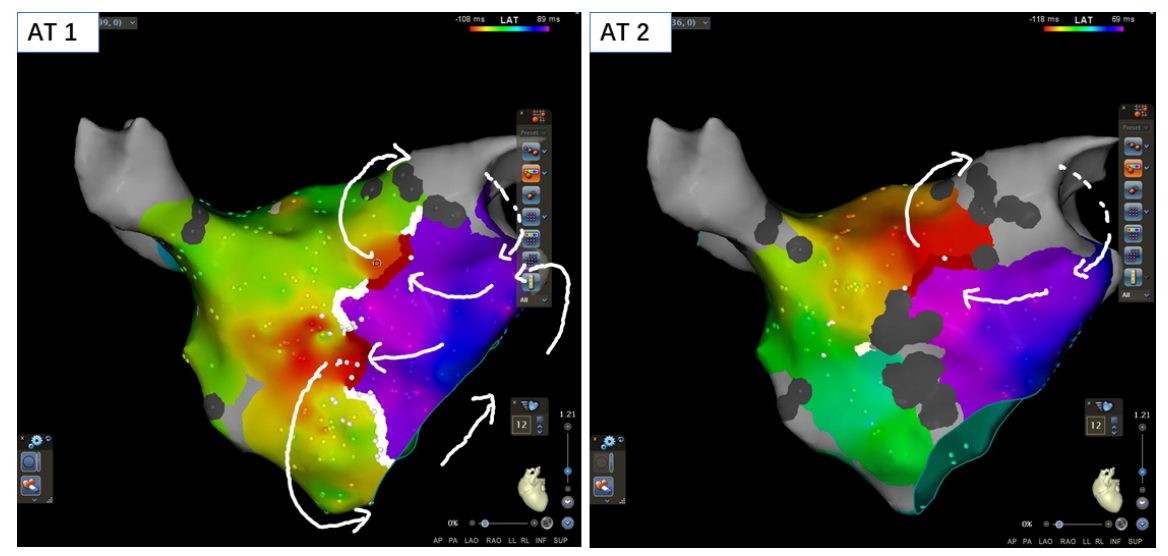

Fig. 4 